\title{
Disseminated mucormycosis presenting with lower extremity weakness
}

\author{
W.R. von Pohle
}

\begin{abstract}
Disseminated mucormycosis presenting with lower extremity weakness. W.R. von Pohle. (C)ERS Journals Ltd 1996.

ABSTRACT: Mucormycosis is an uncommon, but important opportunistic fungal infection. The most commonly reported areas of infection are pulmonary, rhinocerebral and cutaneous. It has only rarely been reported to cause peripheral neurological symptoms.

This report presents the case of a patient who presented with ascending paralysis due to pulmonary mucormycosis. The presentation is believed to be due to a lesion which abutted the vertebral column and caused infarction of the spinal cord. Eur Respir J., 1996, 9, 1751-1753.
\end{abstract}

\begin{abstract}
Correspondence: W.R. von Pohle, Division of Pulmonary and Critical Care, Loma Linda University Medical Center, Room 1521, 11234 Anderson, Loma Linda, California 92354, USA

Keywords: Fungal, mucormycosis, paralysis, pneumonia, respiratory failure

Received October 24 1995, Accepted after revision February 91996
\end{abstract}

The agents of mucormycosis are classified in the order Mucorales, one of the three orders of the class Zygomycetes. The mycelium of these fungi is composed of broad, nonseptate hyphae with right-angled branching, which are the morphological features displayed in the tissues of an infected host. The hyphae of Mucorales often invade the walls and lumen of blood vessels. Thrombosis and resultant infarction of surrounding tissue ensues. Species of Rhizopus, Rhizormucor and Cunninghamella are the most common, but species of Apophysomyces, Saksenaea, Mucor and Absidia occasionally cause mucormycosis. The moulds are rarely septate, have hyphae of uneven diameter, and range $6-50 \mu \mathrm{m}$ in width $[1,2]$. This is the case of a patient who presented with ascending paralysis, new onset diabetes mellitus, and a necrotizing pneumonia.

\section{Case Report}

A 43 year old man without previous medical or surgical illness developed an upper respiratory tract infection and was treated with trimethoprim-sulphamethoxazole. Approximately 2 weeks later, his symptoms recurred. During this episode, his temperature rose to $39.5^{\circ} \mathrm{C}$ with associated chills and production of green, mucopurulent sputum. At this time, he was prescribed 2 weeks of clarithromycin. One week into treatment, he developed left scapular and shoulder pain which was treated with naproxen. His appetite decreased, he had increasing thirst, and his weight decreased from 64 to $58 \mathrm{~kg}$.

Six weeks after first becoming ill, he attended an emergency room because of malaise. At this time, examination disclosed a thin, ill-appearing man. His temperature was $37^{\circ} \mathrm{C}$, pulse 100 beats $\cdot \mathrm{min}^{-1}$, respiratory rate 14 breaths $\cdot \mathrm{min}^{-1}$, and blood pressure $122 / 74 \mathrm{mmHg}$. His lower extremities had decreased strength, bilaterally, with preserved sensation. White blood cell count was $25.7 \times 10^{9}$ cells $\cdot \mathrm{L}^{-1}$, serum glucose level was $599 \mathrm{mg} \cdot \mathrm{dL}^{-1}$, and amylase $945 \mathrm{U} \cdot \mathrm{dL}^{-1}$. Arterial blood gas analysis on room air showed a $\mathrm{pH}$ of 7.01 , arterial oxygen tension $\left(\mathrm{Pa}, \mathrm{O}_{2}\right)$ of $13.3 \mathrm{kPa}(100 \mathrm{mmHg})$, and arterial carbon dioxide tension $\left(P \mathrm{a}, \mathrm{CO}_{2}\right)$ of $1.6 \mathrm{kPa}(12 \mathrm{mmHg})$. Urine and serum ketones were positive. Treatment for diabetic ketoacidosis and pancreatitis was initiated. Chest radiography demonstrated left upper lobe opacifications. A sputum Gram stain revealed Gram-positive cocci and many white blood cells with intracellular organisms. A lumbar puncture was performed and showed normal opening pressure. There were 453 white blood cells $\mu \mathrm{L}^{-1}$ of which $90 \%$ were polymorphonucleates, blood protein was 172 $\mathrm{mg} \cdot \mathrm{dL}^{-1}$, glucose was $133 \mathrm{mg} \cdot \mathrm{dL}^{-1}$, and evaluation for bacterial antigens negative. Erythromycin and cefotaxime were initiated for suspected community-acquired pneumonia. Bronchoscopy was performed and no abnormalities identified on washing or brushing samples. Evaluation for fungal organisms, mycobacterium species, and Pneumocystis carinii was negative. Over the next week, the lower extremity weakness progressed to paralysis and the upper extremities became weak. The patient also developed progressive respiratory insufficiency requiring mechanical ventilation.

At this time, he was transferred to our department. Mechanical ventilation for hypoxic respiratory failure continued, as well as treatment for diabetic ketoacidosis, pancreatitis and pneumonia. Chest radiographs now demonstrated a cavitary lesion in the left lung apex (fig. 1). The day after transfer, computerized tomography (CT) of the chest was performed with needle-guided biopsy for histology and microbiological evaluation. Magnetic resonance imaging of the spine (fig. 2) demonstrated a paraspinal cavitary lesion of the left upper lung and enhancement of the spinal cord at the T3-T4 level; there was no evidence of intraspinal or epidural fluid collections. In $24 \mathrm{~h}$, a culture from the CT guided aspiration demonstrated organisms consistent with Rhizopus species, which were sensitive on culture to amphotericin B and resistant to azole agents. Amphotericin B was initiated at $1 \mathrm{mg} \cdot \mathrm{kg}^{-}$ ${ }^{1}$ q.d. Over the next 2 days, the patient continued to 


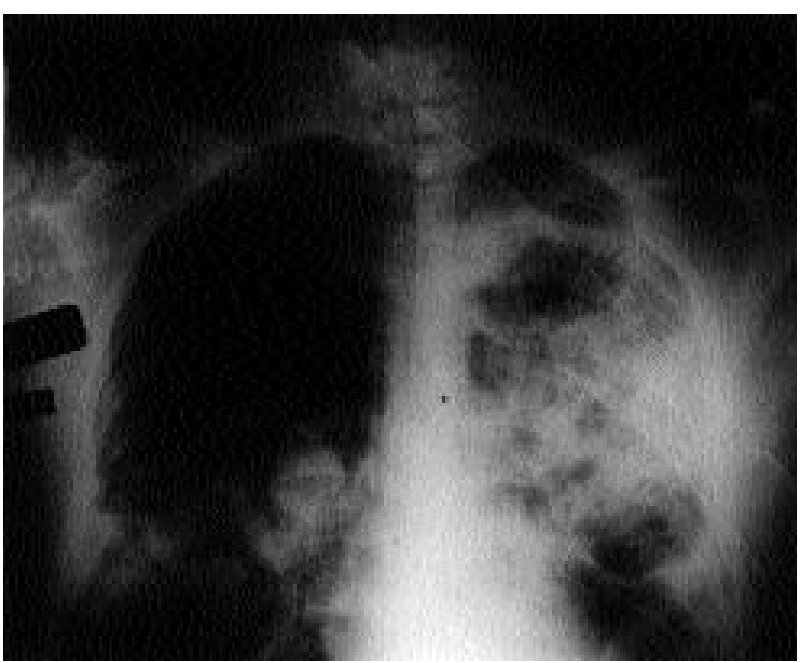

Fig. 1. - Chest radiograph showing bilateral cavitary pneumonia.

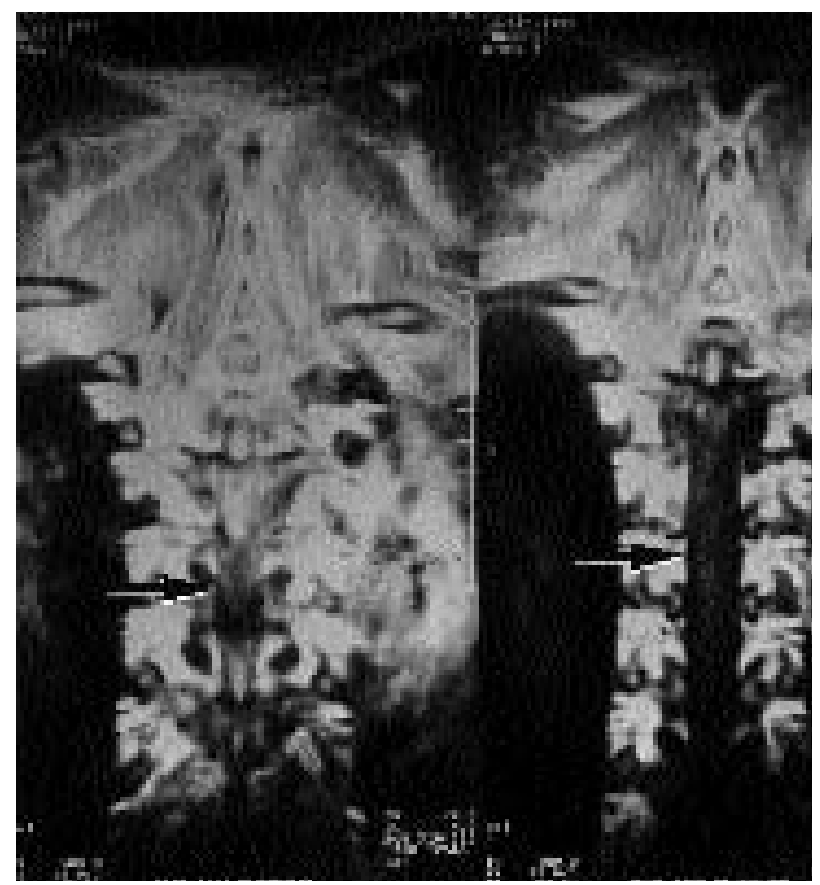

Fig. 2. - Magnetic resonance imaging showing cavitary pneumonia and oedema and enhancement of the thoracic spinal cord (arrow) at the T3-T4 level.

deteriorate with development of multiorgan dysfunction and ensuing death.

At necropsy, disseminated phycomycosis-rhizopus species with severe bilateral necrotizing cavitary pneumonia, bilateral pleural effusions, and direct extension of the left upper lobe abscess to the vertebral bodies at the T3-T4 level was noted. There was an associated purulent leptomeningitis of the spinal canal at levels T3-T8. At the T3 level, there was transverse necrosis of the spinal cord with Rhizopus organisms within feeding blood vessels. In addition, pyelonephric abscesses in the left kidney with mycelial elements were identified.

\section{Discussion}

Rhizopus and Rhizomucor species are ubiquitous. Infection is largely confined to patients with serious preexisting disease [3]. Mucormycosis originating in the paranasal sinuses and nose occurs predominantly in patients with poorly-controlled diabetes mellitus [4]. Pulmonary disease is most commonly seen in patients with leukaemia, lymphoma, or severe granulocytopenia. Most patients have been receiving corticosteroid and antibiotic treatment. Patients with organ transplantation, haematological malignancy, those receiving long-term deferoxamine, and those with acquired immune deficiency syndrome (AIDS) are predisposed to mucormycosis of either the sinuses or lungs [2]. Gastrointestinal mucormycosis has been reported with increased frequency in uraemia, severe malnutrition, and diarrhoeal diseases. Infection is acquired from the environment; person to person transmission is not reported. In all disease forms, vascular invasion by hyphae is predominant. Ischaemic and haemorrhagic necrosis is the predominant histological finding [1]. Repeated cultures of involved areas are frequently negative, and successive biopsies are often required.

Pulmonary mucormycosis is a progressive, severe pneumonia, accompanied by high fever and toxicity. The necrotic centre of large infiltrates may cavitate [2, 5]. Haematogenous spread to other areas of the lung and chest $[6,7]$, as well as to the brain and other organs, is common. Patients usually present with persistent fever, despite treatment with broad-spectrum antibiotics. Survival beyond 2 weeks with pulmonary disease is unusual. Haematogenous dissemination can originate from the lung, paranasal sinuses, or gastrointestinal tract. Sites of dissemination include, the brain, spleen, kidney, heart, liver, pancreas and omentum $[1,8]$.

Chest radiographic abnormalities of mucormycosis include: nodular, lobar, or wedge-shaped opacities; mediastinal widening; miliary pattern; cavitation; fungus ball; and pleural effusion. With such wide radiographic variability, a diagnosis cannot be made or excluded on radiographic grounds alone $[6,9]$. Computerized tomographic findings of the chest have only rarely been reported [7], and, likewise, are not diagnostic.

The hallmarks of treatment are early diagnosis, systemic antifungal therapy, and aggressive surgical debridement [2]. Control of the underlying disease is essential. The antifungal agent of choice is amphotericin B, which is administered until disease progression is halted. The minimum dose of amphotericin B is $1 \mathrm{mg} \cdot \mathrm{kg}^{-1}$ q.d. after a $1.0 \mathrm{mg}$ test dose [6]. Liposomal formulations of amphotericin $\mathrm{B}$ may allow for more aggressive medical therapy and have shown an improvement in outcome [10].

This case demonstrates a new finding, where mucormycosis extended from the lung to direct involvement of the vertebral column and spinal cord. The initial consideration was for an ascending paralysis following a viral upper respiratory tract infection. On reviewing the literature, no previous cases of this type were found. Even when early vigilance and aggressive therapy are applied, the mortality remains high for mucormycosis.

\section{References}

1. Kwon-Chung KJ, Bennett JE, eds. Mucormycosis. In: Medical Mycology. Philadelphia; Lea and Febiger, 1992; pp. 524-559.

2. Bigby TD, Serota MD, Tierney LM, Matthay MM. Clinical spectrum of pulmonary mucormycosis. Chest 1986; 89: 435-439. 
3. Berns JS, Lederman MM, Greene BM. Nonsurgical care of pulmonary mucormycosis. Am J Med Sci 1984; 287: 42-44.

4. Garrison MW, Campbell RK. Identifying and treating common and uncommon infections in the patient with diabetes. Diabetes Educ 1993; 19: 522-531.

5. Dykhuizen RS, Kerr KN, Soutar RL. Air crescent sign and fatal haemoptysis in pulmonary mucormycosis. Scand J Infect Dis 1994; 26: 498-501.

6. Tedder M, Spratt JA, Anstadt MP, Hegde SS, Tedder SD, Lowe JE. Pulmonary mucormycosis: results of medical and surgical therapy. Ann Thorac Surg 1994; 57: $1044-1050$
7. Majid AA, Yii NW. Granulomatous pulmonary zygomycosis in a patient without underlying illness: computed tomographic appearances and treatment by pneumonectomy. Chest 1991; 100: 560-561.

8. Helenglass G, Elliot JA, Lucie NP. An unusual presentation of opportunistic mucomycosis. BMJ 1981; 282: 108-109.

9. Rubin SA, Chaljb G, Winer-Muram HT, Flicker S. Pulmonary zygomycosis: a radiographic and clinical spectrum. J Thorac Imag 1992; 7: 85-90.

10. Hay RJ. Liposomal amphotericin B, AmBisome. J Infect 1994; 28 (Suppl. 1): 35-43. 